\title{
Liderazgo Autentico
}

\author{
Martha de Jesús Guerrero Guzmán ${ }^{1}$ \\ Alicia Cristina Silva Calpa ${ }^{2}$ \\ Jorge Briceño López ${ }^{3}$
}

\section{Resumen}

El tema del liderazgo es de gran importancia en las organizaciones para el manejo de los grupos de trabajo y ante los retos que enfrentan, las nuevas modalidades de liderazgo deben responder a las necesidades de diseño para el cambio, la diversidad, la innovación, asuntos éticos y la respuesta a esas necesidades ha motivado el estudio y análisis de nuevos enfoques como el liderazgo auténtico.

En el presente artículo se revisaran diferentes aspectos relacionados con el liderazgo auténtico, inicialmente se analizará el concepto de autenticidad y de liderazgo auténtico, se presentarán las bases de la Teoría del Desarrollo Auténtico para centrarnos después en cuatro temas importantes como son el Desarrollo del Liderazgo Auténtico, las características de los líderes auténticos, el liderazgo en equipo, el papel del líder con su grupo de trabajo y finalizamos con algunas orientaciones para la dirección de equipos de trabajo.

Palabras claves: Liderazgo auténtico, desarrollo, equipo, autenticidad.

\footnotetext{
${ }^{1}$ Administradora de empresas, Especialista en Pedagogía. Mg. en Administración de Empresas. Universidad Nacional Abierta y Distancia UNAD. martha.guerrero@unad.edu.co.

${ }^{2}$ Administradora de empresas, Especialista en Pedagogía. Mg. Sistemas de Calidad y Productividad. Universidad Nacional Abierta y Distancia UNAD. alicia.silva@unad.edu.co.

${ }^{3}$ Economista, Especialista, Mg. En Administración de Empresas. Universidad Nacional Abierta y Distancia UNAD. jorge.briceno@unad.edu.co.
} 


\section{Introducción}

Sobre el concepto de liderazgo existen diversas definiciones producto de los enfoques, teorías y métodos que se han estudiado, el liderazgo es una característica dinámica que se sucede a través del tiempo y donde están implicados y correlacionados el líder, los seguidores y el momento o la situación en la que se ejerce el liderazgo. El liderazgo se relaciona directamente con la influencia que tiene el líder sobre el grupo o sus subordinados para la obtención de una meta u objetivo común y se considera un fenómeno grupal porque pierde efecto si sale de ese contexto y el presente ensayo pretende profundizar sobre el liderazgo auténtico.

El liderazgo auténtico es uno de los modelos que ha tenido gran acogida en las diferentes organizaciones y para Salanova, Martínez y Llorens (2000) definen el estudio de la Psicología Organizacional Positiva como el estudio científico del funcionamiento óptimo de las personas, los grupos de trabajo y las organizaciones, así como el intento de optimizar y potenciar la calidad de vida laboral.

\section{El concepto de autenticidad.}

Para Avolio y Gardner (2005), el concepto de autenticidad que refiere a ser fiel a uno mismo tiene una larga tradición que se remonta a los antiguos griegos y pasa por la psicología humanista y este concepto viene despertando cada vez más interés en el campo aplicado y académico que reclamaron la existencia de un nuevo tipo de liderazgo por diferentes razones de una parte, la crisis económica, algunos comportamiento de líderes políticos y del sector financiero al momento de afrontar situaciones que puso de manifiesto la importancia de los aspectos éticos y morales del liderazgo y de la otra el auge de la 
Psicología positiva sobre aspectos de autorrealización que relacionaban tanto al líder como a las que trabajan con él.

\section{Concepto de Liderazgo auténtico}

Gil, Alcover y Sánchez (2011) quien cita a (Avolio et al., 2009, p. 423) definen el liderazgo auténtico como un patrón de conducta de liderazgo transparente y ética, que enfatiza la disposición a compartir la información necesaria para la toma de decisiones, a la vez que acepta las aportaciones de los seguidores. Para Avolio y Gardner (2005) los líderes auténticos son individuos profundamente conscientes de sus valores y creencias, de cómo se comportan y, a su vez, de cómo son percibidos por los demás y reconocen y valoran las diferencias individuales y son capaces de identificar los talentos o cualidades positivas de las personas a su cargo y ayudarles a fortalecerlas.

Como resultado, estos líderes son capaces de incrementar la motivación, el compromiso y la satisfacción de sus seguidores a través de la creación de identificación personal e identificación social con la organización y en definitiva, para Avolio y Gardner (2005), el liderazgo auténtico es más genérico que otros tipos de liderazgo y constituye una base sobre la que otros aspectos positivos del liderazgo pueden tener lugar.

\section{Bases de la Teoría del Desarrollo del Liderazgo Auténtico}

Las bases de la Teoría del Desarrollo del Liderazgo Auténtico según (Avolio y Gardner, 2005) son tomadas en primer lugar del Capital Psicológico Positivo que definen la confidencialidad, el optimismo, la esperanza y la resiliencia como aquellas capacidades de los lideres auténticos que permiten desarrollar individuos y equipos y ayudan a florecer y prosperar organizaciones y comunidades, en segundo lugar por la autoconsciencia del 
líder en cuanto a valores, identidad, emociones, objetivos y metas, en tercer lugar por la autorregulación del líder cuando se alinean sus valores con sus intenciones y acciones, en cuarto lugar con la perspectiva moral positiva que tiene que ver con la ética y transparencia del líder en la toma de decisiones, en quinto lugar con el contexto organizacional cuando el líder es capaz de propiciar un entorno y climas organizacionales que permitan un continuo aprendizaje y crecimiento (Gardner et al., 2005; Luthans \& Avolio, 2003).

En sexto lugar el desarrollo de los seguidores que contribuye a su bienestar y al logro de un verdadero rendimiento que se mantiene en el tiempo, en séptimo lugar se relaciona con los procesos y conductas de liderazgo que conducen a incrementar la autoconsciencia y autorregulación de sus seguidores en cuanto a sus valores, identidad y emociones, equilibrando y reduciendo los sesgos en el proceso de la información y por último el rendimiento sostenible y mantenido en el tiempo.

\section{Desarrollo del Liderazgo Auténtico}

Para Gil, Alcover y Sánchez (2011), en los últimos años ha aumentado el interés por el estudio de la relación entre liderazgo y ética basados por el papel que jugaron distintos líderes organizacionales en las crisis financieras y el concepto de liderazgo auténtico surge de la distinción entre liderazgo transformacional auténtico, que muestra un carisma socializado orientado al servicio de los colaboradores, y pseudoliderazgo, que revela un carisma personal orientado al beneficio personal del líder.

\section{Características de los líderes auténticos}

Las cuatro características generales de los líderes auténticos que identifican Shamir \& Eilam (2005), se refieren a ser sinceros consigo mismos, están motivados por 
convicciones personales frente al status, honor u otro tipo de beneficios, son originales y dirigen desde su punto de vista personal y para Avolio (2003) los líderes auténticos saben dónde están ubicados, mantienen su rumbo y lo comunican a los demás a través de sus palabras y de sus actos cuando se trata de valores y creencias importantes.

Otra característica de estos líderes es su imparcialidad para el proceso de la información y las relaciones con sus seguidores se basan por los altos niveles de respeto, afecto, confianza y a reforzar la efectividad de su propio enfoque del auténtico liderazgo.

\section{Liderazgo de Equipo}

Para Gil, Alcover y Sánchez (2011) al aumentar la presencia de los equipos en las organizaciones, la investigación se ha centrado en el impacto del liderazgo sobre el rendimiento de equipo y la influencia que ejerce el líder individual sobre sus colaboradores a través de relaciones interpersonales ante la dificultad de que un líder externo pueda desempeñar exitosamente todas las funciones de liderazgo.

Cuando el trabajo se basa en el conocimiento, es importante que aquellos profesionales tengan la suficiente autonomía para desempeñar funciones de liderazgo en las organizaciones, Alcover y Tabernero (2010) plantean como el liderazgo promueve los procesos de los equipos en cuanto a cómo se consigue integrar las actividades de equipo, crear un buen clima de trabajo y el desarrollo de un aprendizaje colectivo.

Según Gil, Alcover y Sánchez (2011) quien cita a (Day, Gron y Salas, 2004) el liderazgo de equipo puede entenderse de dos formas diferentes de una parte como aquellos atributos, habilidades y conductas que aportan los miembros al equipo como son el carisma, la integridad y la proactividad y que operan como insumos que influyen en los procesos y 
el rendimiento del equipo y de otra parte como resultado de procesos de equipo como el caso del aprendizaje grupal, facilitando la adaptación y el rendimiento del equipo a través de las diferentes etapas de su desarrollo. Para el autor ambos enfoques resultan esenciales para entender el liderazgo de equipo.

El primer enfoque nos muestra la importancia del funcionamiento del liderazgo en los grupos de trabajo a partir de la contribución del líder sobre los procesos y resultados que se generen pues es claro que un equipo puede poseer los conocimientos necesarios para desempeñar su trabajo, pero es el líder definiendo metas, organizando el equipo, motivándolo y apoyándolo quien puede colaborar a conseguir los objetivos propuestos.

Sobre este enfoque Gil, Alcover y Sánchez (2011) destacan algunos modelos importantes sobre equipos de trabajo, un primer modelo señala cómo los líderes pueden influir en el aprendizaje y desarrollo del equipo en diferentes etapas del trabajo realizando inicialmente funciones de motivación para familiarizarse con el grupo, luego funciones consultivas que se refieren a la tarea a realizar y por último funciones educativas que son orientadas a la reflexión, este modelo fue propuesto por Hackman y Wageman (2005).

Un segundo modelo plantea como as competencias de liderazgo influyen en el rendimiento del equipo a través de procesos grupales cognitivos, motivacionales, afectivos y de coordinación proponiendo para los procesos cognitivos competencias de liderazgo que tiene que ver con los modelos mentales compartidos y el procesamiento de la información colectiva y motivación para los miembros del grupo, para los procesos motivacionales la cohesión y eficacia colectiva a través de la planificación, la motivación y la retroalimentación y para los procesos afectivos utilizar herramientas para el control del 
conflicto y emociones y por último para los procesos de coordinación se debe buscar alinear las capacidades de los miembros con sus roles, ofrecer estrategias claras, supervisar los cambios en el ambiente, dar retroalimentación y reorganizar, este modelo fue planteado por Zaccaro, Heinen y Shuffler (2009)

En general los modelos proponen que el impacto del liderazgo de equipo se basa en proveer mayor interconectividad, integración y coherencia entre los miembros. El rol fundamental del líder, además de establecer la dirección y gestionar operaciones del equipo, consiste en desarrollar la capacidad y pericia de liderazgo de los miembros de equipo.

\section{El papel del líder autentico con su grupo de trabajo}

El liderazgo auténtico está relacionado con la identificación de los seguidores con el líder y su grupo (Avolio et al, 2004), los líderes son capaces de incrementar en sus seguidores la identificación social con su grupo, creando un alto sentido moral y expresando altos niveles de honestidad e integridad en las interacciones, para (Hogg, 2001), las características del líder como miembro del grupo juega un rol importante para la eficacia del liderazgo con sus seguidores, grupo u organización.

En grupos con alta identificación grupal, los miembros más prototípicos se convertirán más fácilmente en líderes efectivos e incluso más aún, si emplean sus credenciales prototípicas (Moriano et al, 2009). Sin embargo, un nivel mínimo de identificación de los seguidores con el grupo es una condición necesaria para traducir prototipicidad en eficacia del liderazgo; a partir de este nivel mínimo, incrementos en el nivel de identificación grupal se asume que incrementan los efectos de la prototipicidad del líder (Ullrich et al; 2009). 


\section{Equipos de trabajo}

Para la efectividad de los equipos de trabajo y el buen funcionamiento de las organizaciones el liderazgo ha tomado crucial importancia, Gil, Alcover y Sánchez (2011) expresan que la investigación sobre liderazgo ha conseguido importantes avances desde los años 30 en que se inició su estudio, en los años 70 ante la falta de avances significativos se llegó a proponer el abandono del propio concepto de liderazgo, mientras que en los años 80 se pasó a una fase de entusiasmo en la que se desarrollaron los denominados nuevos modelos de liderazgo y proliferaron las investigaciones.

Citando a Burke y Cooper, 2006 los autores expresan que hechos importantes como la globalización, las nuevas tecnologías de la comunicación y los cambios económicos y sociales plantean a las organizaciones nuevos roles y funciones de liderazgo, y han vuelto a impulsar al alza su interés investigarlo.

Para los autores, la necesidad de afrontar nuevos retos exige a los líderes adoptar un papel complejo, capaz de compaginar la continuidad con el cambio y la innovación, y que a la vez esté sólidamente fundado en principios éticos y valores sociales. Asimismo, el escenario derivado de la globalización requiere ejercer un liderazgo global e integrador, sensible a las diferencias cultura-les. También, la creciente complejidad e incertidumbre de las situaciones empresariales actuales y el trabajo intensivo en conocimiento hacen inviable la actuación de un único líder y requieren equipos multiprofesionales, con autonomía para trabajar y un liderazgo compartido que emerja del propio equipo. Finalmente, las nuevas formas de trabajo, como los equipos virtuales o equipos distribuidos, modifican las 
relaciones tradicionales del líder con los colaboradores, requiriendo un nuevo tipo de liderazgo a distancia y con delegación de funciones.

\section{Conclusiones}

Los nuevos contextos y retos a los que se enfrentan las organizaciones requieren reexaminar y ampliar los modelos tradicionales de liderazgo y en este escrito pretendíamos analizar los efectos del liderazgo a nivel de grupo o equipo, puesto que es en entornos grupales donde ocurren estos procesos.

El análisis del liderazgo auténtico nos muestra que este tipo de liderazgo facilita el desarrollo de los grupos de trabajo a la hora de alcanzar los objetivos de las organizaciones. La revisión de esta nueva modalidad de liderazgo autentico nos permite concluir que es de gran importancia para que los directivos puedan reflexionar sobre los posibles abusos de poder y efectos indeseados del liderazgo pues los autores que estudiaron el tema proponen la defensa de códigos éticos y valores relacionados con el carisma socializado y estas sugerencias resultan útiles porque representan guías para el diseño de programas de formación y para la elaboración de taxonomías de com-petencias de liderazgo.

El desarrollo del liderazgo auténtico es un proceso que implica un cambio en el que los seguidores y los líderes incrementan su autoconsciencia, y establecen relaciones abiertas y transparentes, sinceras y genuinas, las cuales pueden ser en parte formuladas y moldeadas por intervenciones planificadas.

\section{Fuentes Bibliográficas:}

Avolio, B; Gardner, W. (2005) Authentic leadership development: Getting to the root of positive forms of leadership The Leadership Quarterly 16) 315-338\} 
Gardner, W.L; Avolio, B. J; Luthans, F; May, D.R; Walumbwa, F.(2005) “Can you see the real me?" A self-based model of authentic leader and follower development. The Leadership Quarterly 16 343-372

Gil, F.; Alcover, C.M.; Rico, R., \& Sánchez, M. (2011). Nuevas Formas de Liderazgo en Equipos de Trabajo. Papeles del psicólogo, vol. 32, núm. 1, enero-abril, 2011, pp. 38-47 consejo general de colegios oficiales de psicólogos. Madrid, España.

Hogg, M. A. (2001). A social identity theory of leadership. Personality and Social Psychology Review, 5, 184-200.

Moriano, J.A; Topa, G; Lévy, J.P. (2009) Leadership in Nonprofit Organizations of Nicaragua and El Salvador: A Study from the Social Identity Theory. The Spanish Journal of Psychology. Vol. 12, No. 2, 667-676

Salanova, M. Martínez, I. y Llorens, S. (2000) Psicología Organizacional Positiva. En Palací, J.F (2008) Psicología de la Organización. Ed Pearson. España, pp 349-376

Shamir, B., \& Eilam, G. (2005). bWhat's your story?Q: A life-stories approach to authentic leadership development. The Leadership Quarterly.

Ullrich, J; Christ, O; van Dick, R. (2009) Substitutes for Procedural Fairness: Prototypical Leaders Are Endorsed Whether They Are Fair or Not Vol. 94, No. 1, 235-244 